\title{
Modeling and Analysis of Physical Human-Robot Interaction of an Upper Body Exoskeleton in Assistive Applications
}

\author{
Simon Christensen ${ }^{1}$ Xuerong $\mathrm{Li}^{1}$ Shaoping Bai ${ }^{1}$ \\ ${ }^{1}$ Department of Materials and Production, Aalborg University, Aalborg 9220, Denmark. E-mail: \\ $\{$ sic, $x l$, shb $\} @ m p . a a u . d k$
}

\begin{abstract}
Portable exoskeletons can be used to assist elderly or disabled people in their daily activities. The physical human-robot interaction is a major concern in exoskeleton development for both functioning properly and interacting safely and comfortably. Using a model of the human musculoskeletal system and the exoskeleton can help better understanding, estimating and analyzing the physical human-robot interaction. In this paper, a model comprising the biomechanics of human upper body and the dynamics of a 4-DoF exoskeleton, named UB-AXO, is developed and used to study the physical human-robot interaction. The human-exoskeleton model is able to estimate effect of physical human-exoskeleton interaction, such as muscle activity, and energy consumption and human joint reaction forces, when performing cooperative motions with the exoskeleton. The model development is described and subsequently two simulation studies of typical activities of daily living are conducted to analyze and evaluate the performance of the UB-AXO. The simulation results demonstrate that the UB-AXO is able to reduce muscle loading and energy consumption, while maintaining a safe physical human-exoskeleton interaction.
\end{abstract}

Keywords: Physical Human-Robot Interaction, Biomechanical Modeling, Assistive Exoskeleton, Energy Exchange in pHRI, Overhead Reaching Tasks

\section{Introduction}

In the field of wearable robotics, exoskeletons are becoming more and more relevant in domains such as healthcare and industry Sylla et al. (2014); Bai et al. (2018). An exoskeleton is a robotic suit that is capable of producing supplementary muscular functions to its user, by either powered elements (e.g. electric motors) or passive elements (e.g. springs). The exoskeleton enables the user to lift a greater load or compensate for a lack of strength Bock et al. (2021); Gull et al. (2020); Pacifico et al. (2020). In general, exoskeletons are designed to transfer mechanical power to a specific set of human joints, e.g. elbow flexion/extension, by imitating the kinematics of the given body limbs.
Hence, assisting human movements using exoskeletons requires consideration of the contribution of both the human biomechanics and exoskeleton mechanics to the assisted joint. While exoskeletons have the potential to improve the users functionalities and strength, a safe and comfortable interaction with human limbs is a major design and control challenge. The operations of the exoskeleton must be properly coordinated and adapted to the human since unintended interaction can lead to injuries on the human.

Many factors affect the physical human-robot interaction (pHRI) Bicchi et al. (2008); De Santis et al. (2008); Heinzmann and Zelinsky (2003); Davis et al. (2020). One is the transfer of force from an exoskeleton to the human body and consequentially the energy 
flow. The forces or torques exerted by the robot onto the human should be delivered as needed. This means that the magnitude of the assistive force/torque has to be specified properly from the perspective of control. Moreover, the interaction should be soft and compliant and most importantly must never exceed the human pressure tolerance.

Another issue in the pHRI is the alignment between human joints and the counterparts of an exoskeleton. Ideally, the exoskeleton should be well aligned with human limbs, but this is quite difficult to achieve, especially for the upper-body exoskeletons Gopura et al. (2016); Schiele and van der Helm (2006); Zhou et al. (2015); Nf et al. (2019). One reason is that the exact location of the human joint axes is not possible to know, simply because they are covered up by human skin, muscles and tissue. Moreover, biological joints are not ideal mechanical joints (like hinge or ball and socket joints), but are rather complex joints' surface geometries (bone on bone joints). As a result, the equivalent rotational joint axes tend to shift during motion. In addition, attachments of exoskeletons on human limbs are not rigid, meaning that slippage between the exoskeleton and the human limb can occur during operation. Consequently, these issues are likely to cause misalignment between the exoskeleton joint and the human joint in the order of a few centimeters Schiele and van der Helm (2006), which can generate undesired reaction forces in the human joint, leading to uncomfortable and possibly painful interaction with the exoskeleton Schiele (2008).

Biomechanical modeling of human-exoskeleton systems is an effective approach to address the aforementioned issues in pHRI. In previous works, musculoskeletal models have been used to analyze the physical interaction between the coupled human-exoskeleton systems Agarwal et al. (2016); Zhou et al. (2017); Bai and Rasmussen (2011); Narayanan et al. (2009). Using advanced musculoskeletal models can gain insight and predict the human response to assistance from exoskeleton. Moreover, using a virtual prototyping environment is less expensive and time consuming than building mock-up models for physical tests.

It is noticed in the models reported, the coupled human-exoskeleton system is usually assumed rigidly connected to the human. This is an oversimplified contact problem, as it cannot simulate the human contact with different attachment. Moreover, the simulations mainly addressed the interaction forces, the power flow is rarely considered.

In this work, a physical human-robot simulation model is developed for a portable upper-body assistive exoskeleton, named UB-AXO. The model includes a biomechanical human subsystem and a mechanical ex- oskeleton subsystem, both integrated for physical assistance simulations. A contact model is utilized for an improved physical human-exoskeleton interaction modeling. The model is developed by virtue of an advanced biomechanical model of the upper body, which is developed through the AnyBody Modeling System (AnyBody Technology A/S, Aalborg, Denmark). Six different case studies are considered in order to assess the performance of the UB-AXO in collaboration with the human user. The developed model allows us to understand the mechanics of the cooperative humanexoskeleton system, and finally to design and control exoskeletons with improved physical human-robot interaction.

\section{A conceptual design of an upper-body assistive exoskeleton (UB-AXO)}

Our interest is the pHRI in assistive exoskeletons, which work cooperatively with human to perform a certain limb movement. Our model is developed for an assistive exoskeleton named UB-AXO. The UB-AXO is an upper-body exoskeleton developed at Aalborg University, Denmark, see Fig. 1. It is designed to assist the user at joint level with activities such as lifting and carrying objects. The UB-AXO has a total of four degrees of freedom, three at the shoulder and one at the elbow joint.

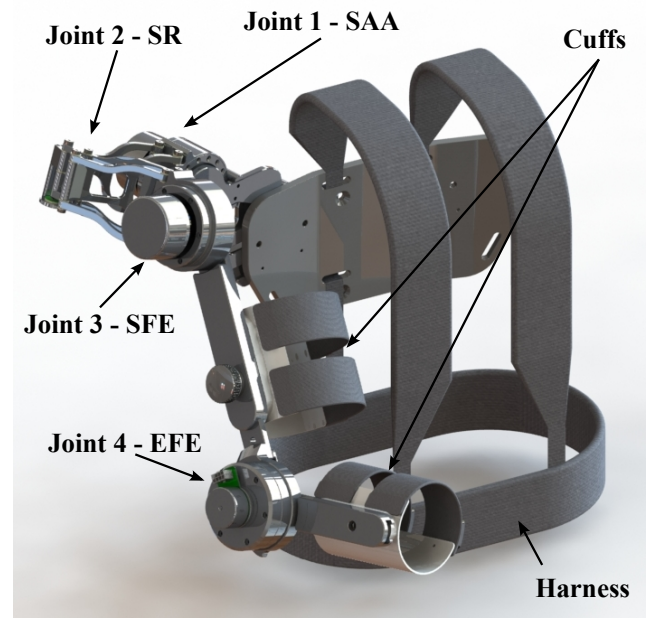

Figure 1: Conceptual design of the UB-AXO

The shoulder mechanism is designed to match the three degrees of freedom of human glenohumeral joint. The shoulder abduction/adduction (SAA) and flexion/extension (SFE) joints are powered, while shoulder internal/external rotation (SR) joint is passively 
supported by a double parallelogram linkage (DPL) Christensen and Bai (2018). The elbow mechanism is a single powered joint that supports flexion/extension of the human elbow (EFE). All active joints are composed by a harmonic gear and brushless DC-motor. The harmonic gear is selected for its back-drivability, which allows the user to move even if the motors are powered off.

The UB-AXO is worn by the user through a torso harness, an upper arm cuff and a forearm cuff. The torso harness is composed by hard back plate fitted with shoulder straps and snap buckle belt for rapidly fitting and easy tightening to the user. Both the upper arm and forearm cuffs consist of a flexible plastics material and are tighten to the limb using velcro straps.

The base of the shoulder mechanism, i.e. the shoulder abduction/adduction joint, and the upper arm link are adjustable to fit the user's body size.

\section{Biomechanical model of the human-exoskeleton system}

The human-exoskeleton system is a cooperative system of the exoskeleton mechanics and the human biomechanics, see Fig. 2. Thus, the model is built with two subsystems, namely, the human and the exoskeleton. The biomechanics of the human upper body is considered through a musculoskeletal model by simulating the movements of the arm and the required muscle activations, while the exoskeleton model incorporates the dynamics and control of the exoskeleton.

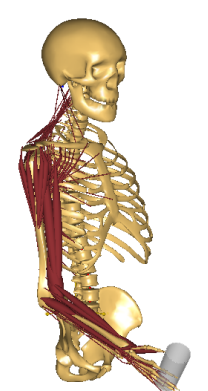

a)

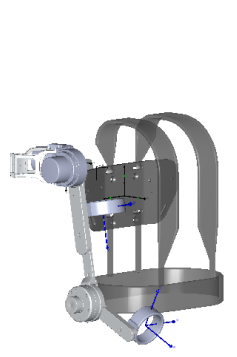

b)

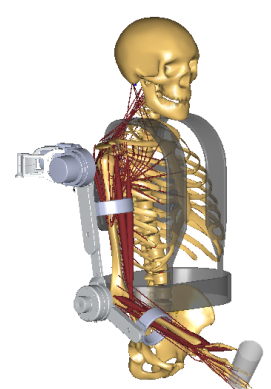

c)
Figure 2: Model of the human-exoskeleton system: a) the human biomechanics, b) the exoskeleton model, c) the cooperative human-exoskeleton system

\subsection{Musculoskeletal model of the human upper body}

The musculoskeletal model of the human upper body is modeled as a multi-body system, where human bones and joints are considered as mechanical links and ideal joints. Muscles are unidirectional actuators that exert forces on the system. Because of the redundancy of muscles in the system, the system is statically indeterminate. Therefore, the muscle recruitment is formulated as an optimization problem, named direct muscle recruitment Rasmussen et al. (2001):

$$
\begin{aligned}
& \min G\left(\mathbf{f}^{M}\right) \\
& \text { s.t. } \mathbf{C ~} \mathbf{f}=\mathbf{d}
\end{aligned}
$$

Here $\mathbf{f}=\left[\mathbf{f}^{M} \mathbf{f}^{R}\right]$ is a vector with all unknown forces, where $\mathbf{f}^{M}$ is an array of the muscle forces and $\mathbf{f}^{R}$ is the reaction forces in the joints. The matrix $\mathbf{C}$ is a coefficient matrix formed from the human anatomy and muscle attachments and finally, the vector $\mathbf{d}$ is an array of the external forces acting on the human. The objective function min $G\left(\mathbf{f}^{M}\right)$ is the muscle recruitment criterion and is usually a polynomial criterion, but soft saturation and $\min / \max$ criterion are also feasible Rasmussen et al. (2001). In this paper, the polynomial criterion is applied:

$$
\min G\left(\mathbf{f}^{M}\right)=\sum_{i}\left(\frac{f_{i}^{M}}{N_{i}^{M}}\right)^{p}
$$

The term $f_{i}^{M} / N_{i}^{M}$ is referred to as muscle activity. Here $N_{i}^{M}$ is a normalization factor or function for the $i$ th muscle, which represents the strength of the muscle. Hence, the stronger the muscle, the larger the normalization factor. The power $p$ represents the synergism between the muscles. In this work, the power of $p=3$ is used, as numerical experiments with it yield good results for submaximal loads. The muscle activities range from 0 to 1 , where 0 is an unloaded muscle and 1 is a fully loaded muscle. The muscle forces are calculated using a three-element Hill-type muscle model, which consists of a contractile element (CE), a parallel elastic element (PE) and a serial elastic element (SE) Winter (2008).

In addition to the muscle activities, we are also interested to find the metabolic cost. The metabolic energy is a common evaluation measure for assistive devices and is often experimentally determined by a VO2 mask, which measures the volume of oxygen consumption by the test subject Rowe (2020). While modeling the individual muscle metabolic energy requires complex formulas Bhargava et al. (2004), which includes 
information on heat dissipation of the muscle, we calculate in this work the human energy consumption from the mechanical work produced by the contractile element of the muscle model, i.e. the active element of the muscle model. The muscle work rate $\dot{W}$ is calculated as:

$$
\dot{W}=f_{C E}\left(l_{C E}, v_{C E}, s\right) v_{C E}
$$

where $f_{C E}$ is the force produced by the contractile muscle element, which is a function of the length $l_{C E}$ and velocity $v_{C E}$ of the element along with the muscle activity $s$. For the muscles, both positive and negative works are possible. When a muscle produces positive work, or concentric work, energy is fed to the musculoskeletal system. On the other hand, when a muscle produces negative work, or eccentric work, energy is extracted from the musculoskeletal system. The contractile element of the muscle cannot store energy, instead the energy is dissipated as heat. Hence, the total human energy consumption is estimated as the absolute value of the external work done on the human bodies:

$$
E=\sum_{i=1}^{N} \int_{t_{i}}^{t_{f}}\left|\dot{W}_{i}\right| \mathrm{dt}
$$

where $N$ is the total number of muscles in the model.

\subsection{Exoskeleton model}

The configuration of the UB-AXO is illustrated in Fig. 3. The shoulder mechanism is composed by the first three revolute joints, which together form a spherical joint, equivalent to the glenohumeral joint of the human shoulder. The forth revolute joint makes up the elbow mechanism. The Denavit-Hartenberg parameters for the UB-AXO are listed in Table 1, where $L_{u}$ is the length of the upper arm and $L_{f}$ is the length of the forearm.

Table 1: Denavit-Hartenberg parameters of the UB$\mathrm{AXO}$

\begin{tabular}{lrrrr}
\hline \hline Link, $i$ & $a_{i}$ & $\alpha_{i}$ & $d_{i}$ & $\theta_{i}$ \\
\hline 1 & 0 & $-\pi / 2$ & 0 & $\theta_{1}$ \\
2 & 0 & $\pi / 2$ & 0 & $\theta_{2}$ \\
3 & $L_{u}$ & 0 & 0 & $\theta_{3}$ \\
4 & $L_{f}$ & 0 & 0 & $\theta_{4}$ \\
\hline \hline
\end{tabular}

The Jacobian of the exoskeleton can be calculated through the velocity propagation from link to link, which yields:

$$
\mathbf{J}=\left[\begin{array}{rrrr}
J_{11} & J_{12} & J_{13} & J_{14} \\
J_{21} & J_{22} & J_{23} & J_{24} \\
0 & J_{32} & J_{33} & J_{34} \\
0 & J_{42} & J_{43} & J_{44} \\
0 & J_{52} & J_{53} & J_{54} \\
1 & 0 & J_{63} & J_{64}
\end{array}\right]
$$

where the elements of the Jacobian are listed in the Appendix A.

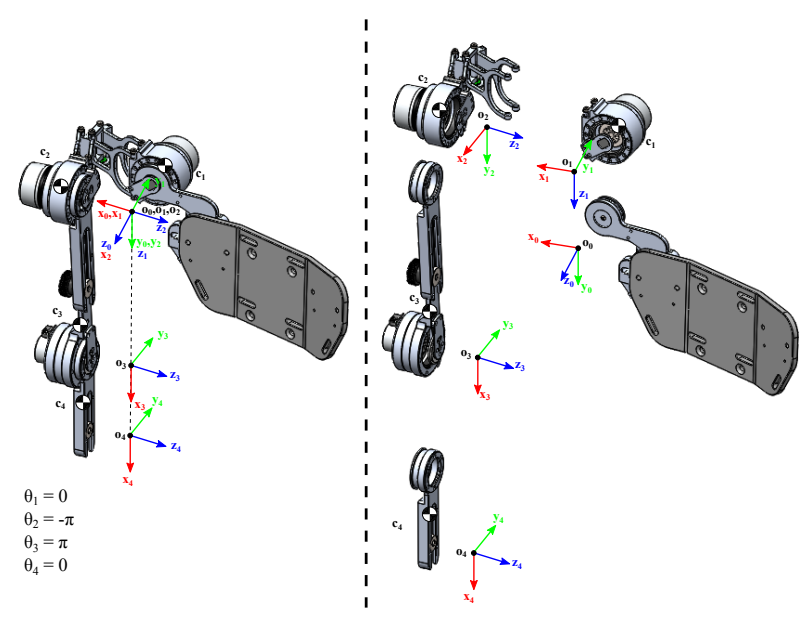

Figure 3: The coordinate systems of the UB-AXO

The inverse dynamics of the exoskeleton is derived using the Euler-Lagrange equation and expressed as:

$$
\mathbf{M}(\boldsymbol{\theta}) \ddot{\boldsymbol{\theta}}+\mathbf{c}(\boldsymbol{\theta}, \dot{\boldsymbol{\theta}})+\mathbf{g}(\boldsymbol{\theta})=\boldsymbol{\tau}
$$

where $\mathbf{M}$ is the mass matrix, $\mathbf{c}$ is a vector with the Coriolis and centrifugal forces, $\mathbf{g}$ is the vector of gravitational forces, $\boldsymbol{\tau}$ is the vector with joint torques and $\boldsymbol{\theta}$ is a vector with the joint angles, as illustrated in Fig. 3.

\subsection{Assistance from exoskeleton}

The UB-AXO is designed to assist the human body at joint levels, i.e. shoulder abduction/adduction and flexion/extension and elbow flexion/extension, while shoulder internal/external rotation is left passive and unassisted. In assistive applications of active joints, the assistive torques are determined through a control strategy that uses inputs from the system.

In this work, a static-load compensation strategy is adopted, as illustrated in Fig. 4. The assistive torque of each active joint $\boldsymbol{\tau}_{\text {ass }}$ is calculated with three parts; an exoskeleton gravity compensation torque $\tau_{\text {exo }}$, a human gravity compensation torque $\boldsymbol{\tau}_{g}$ and torque for external loads $\boldsymbol{\tau}_{l}$.

$$
\boldsymbol{\tau}_{a s s}=\boldsymbol{\tau}_{\text {exo }}+\mathbf{K}\left(\boldsymbol{\tau}_{g}+\boldsymbol{\tau}_{l}\right)
$$




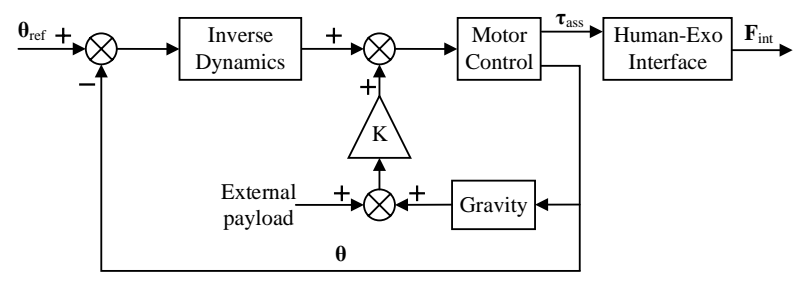

Figure 4: Implementation of the control strategy in the human-exoskeleton model, $\mathbf{F}_{\text {int }}$ standing for assistive interaction force to human limb

where $\mathbf{K}=\operatorname{diag}\left(k_{1}, k_{2}, k_{3}, k_{4}\right)$ is the assistance levels at all joints. It should be noted that $k_{2}$ and the second entry of $\boldsymbol{\tau}_{\text {exo }}$ are equal to zero, as joint 2 in our exoskeleton is a passive joint.

Typically, the mechanical interaction between the human and exoskeleton is addressed through interaction control, such as impedance or admittance control L. Pons (2008). Because accelerations and velocities of the human movement are assumed small, the interaction between the human and exoskeleton can be considered quasi-static and the mechanical interaction is simplified to a gravity compensation of the exoskeleton. Hence, the exoskeleton gravity compensation torque is calculated as:

$$
\boldsymbol{\tau}_{\text {exo }}=\mathbf{g}(\boldsymbol{\theta})
$$

The human gravity compensation torque is based on estimated mass properties of the human. In Winter (2008), the mass of individual body limbs are linked to the total weight of the body, namely, the mass of the upper arm is approximately $2.8 \%$ of the total body mass, while the forearm makes up $1.6 \%$ of the total body mass Winter (2008), that is:

$$
m_{u}=0.028 m_{b}, \quad m_{f}=0.016 m_{b}
$$

As the exoskeleton is presumably kinematically compatible with the human kinematics, the human gravity compensation torques can be calculated from the exoskeleton joint angles.

$$
\boldsymbol{\tau}_{g}=\mathbf{g}_{h}(\boldsymbol{\theta})
$$

where $\mathbf{g}_{h}$ is a vector with the estimated human gravitational forces.

The external load is not directly attached to the exoskeleton, but at the hand of the human. The nature of the external load can be quite complex for a variety of tasks where the human interacts with the environment, e.g. opening a door or pushing an object on a table. Regardless of the complexity of the external load, the Jacobian in Eq. (5) can be used to link the external load at the human hand with the exoskeleton joint torques.

$$
\boldsymbol{\tau}_{l}=\mathbf{J}^{T} \mathbf{F}_{e x t}
$$

where $\mathbf{F}_{\text {ext }}$ is a vector with the external load expressed with respect to the global reference frame.

\subsection{Human-exoskeleton model}

The two subsystems, i.e. the musculoskeletal model of human body and the CAD model of the exoskeleton, are implemented in the AnyBody Modeling System, see Fig. 2. AnyBody Modeling System in its essence is an inverse dynamics simulation software that uses a generic musculoskeletal model of human body to study the internal body loads, i.e. muscle, ligament and joint forces, under different motions and external loads. In this study, the musculoskeletal model is comprised of a human torso and right arm, which is derived from the repository in AnyBody. The exoskeleton model is built in SolidWorks and exported to AnyBody. The motion of the musculoskeletal arm is used to drive the cooperative system. The arm motion is generated from motion capture data of people doing the specific task or set of tasks. For simple tasks, such as an arm curl, the model can be driven by explicit functions for each joint.

As the human musculoskeletal and exoskeleton subsystems are connected via attachments, additional kinematic constraints and a contact model need to be defined for the whole system, as described presently.

\subsection{Kinematic constraints}

The exoskeleton model is connected to the musculoskeletal model at the three ports of power transfer, namely the torso harness, upper arm and forearm cuff. The torso harness is fixed to the torso of the musculoskeletal model by six kinematic constraints, such that the shoulder module is aligned with the human glenohumeral joint. The upper arm and forearm cuffs are attached at the mid point of the upper arm and forearm, respectively. Both cuffs are modeled as cylindrical joints, which add additional eight kinematic constraints. Hence, in total 18 kinematic constraints are used to connect the exoskeleton model to the musculoskeletal model. The UB-AXO has a total of ten degrees of freedom, before it is mounted to human body. The additional 18 kinematic constraints make the whole system kinematically over-determined and there is no unique solution to the kinematics. This problem is solved as an optimization problem, where the constraints are divided into 'hard' and 'soft' constraints. Hard constraints are constraints in the kinematic analysis, which must be fulfilled, while soft constraints are constraints that should be fulfilled as well 
as possible by the optimization algorithm. As a result, small kinematic errors are introduced to the model. Similar effect can be experienced in a 'real' application, where the human and exoskeleton exhibit small relative motions because of the wearer's skin or clothes. All kinematic constraints, in this work, are defined as soft constraints, where the allowable kinematic error for the solver is set to $0.1 \%$.

\subsection{Contact modeling}

The physical interaction between the human and exoskeleton is a contact problem. The assisting torques from the exoskeleton are transmitted to the human musculoskeletal system via contact forces acting between the exoskeleton cuffs and the skin of the human.

The contact forces are determined by calculating the reaction forces between two nodes, a base and a target node, one on the human musculoskeletal body and the other on the exoskeleton. A cylindrical space is defined around the base node. When the target node enters the cylindrical space of the base node, contact is established between the two objects, see Fig. 5. The contact forces are modeled as unilateral normal force and a perpendicular friction force. The friction force is limited by the size of the normal force and the friction coefficient. A set of base nodes are defined on the exoskeleton at the upper arm and forearm cuffs. The nodes form a circular arc with a radius $r$ upon which a total of 12 base nodes are evenly distributed. From anthropometric measurements listed in Peebles and Norris (1999), the inner diameter of the upper arm is estimated to $103 \mathrm{~mm}$ and forearm is estimated to 76 $\mathrm{mm}$, which fits $50 \%$ of the population.

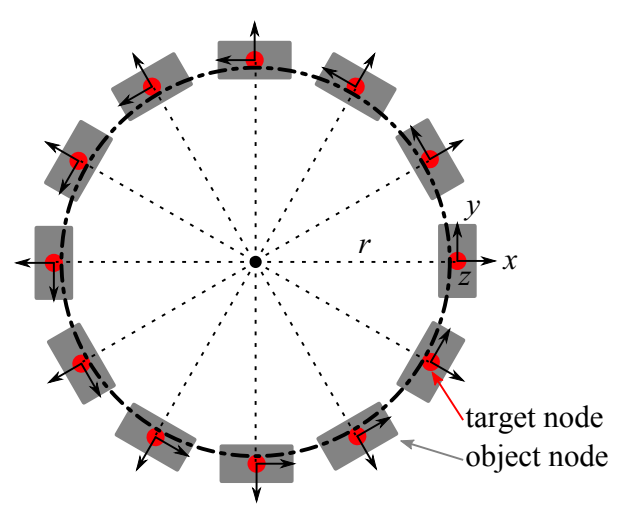

Figure 5: Implementation of contact element for human-exoskeleton interaction forces

Similarly, as for the muscle recruitment, the contact forces have an optimum value, which can be determined through a polynomial criterion. Hence, the cost function in the direct muscle recruitment Eq. (2) is updated to include the contact forces Skals et al. (2016):

$$
\min G\left(\mathbf{f}^{M}\right)=\sum_{i}\left(\frac{f_{i}^{M}}{N_{i}^{M}}\right)^{3}+\sum_{j}\left(\frac{f_{j}^{C}}{N_{i}^{C}}\right)^{3}
$$

where $f_{j}^{C}$ is the contact force and $N_{i}^{C}$ is the optimal contact force.

\section{Simulation studies}

To evaluate the performance of the exoskeleton, two cases are considered. In the first case, the elbow mechanism performs a simple bicep curl, while in the second case, the shoulder mechanism assists an overhead reach task. In both simulations, the motion is generated from explicit functions of the individual joints. The payload in the biceps curl case is $2 \mathrm{~kg}$, while for the overhead reach it is $1 \mathrm{~kg}$. For each case, three studies are simulated. The first study simulates the musculoskeletal model performing the task without the exoskeleton. The second and third studies simulate the musculoskeletal model with the exoskeleton providing $30 \%$ and $50 \%$ assistance, respectively. The assistance level is controlled through the assistance matrix, $\mathbf{K}$, from Eq. (7), where the 30\% (low-level) assistance implies $k_{1}=k_{3}=k_{4}=0.3$ and $50 \%$ (high-level) assistance implies $k_{1}=k_{3}=k_{4}=0.5$.

\subsection{Case 1 - Bicep curl}

In the bicep curl case, the elbow is flexed from $120^{\circ}$ to $30^{\circ}$ and back again over a period of $3 \mathrm{~s}$, see Fig. 6 . Furthermore, the shoulder is slightly flexed to an angle of $5^{\circ}$ to keep the load free from the body.
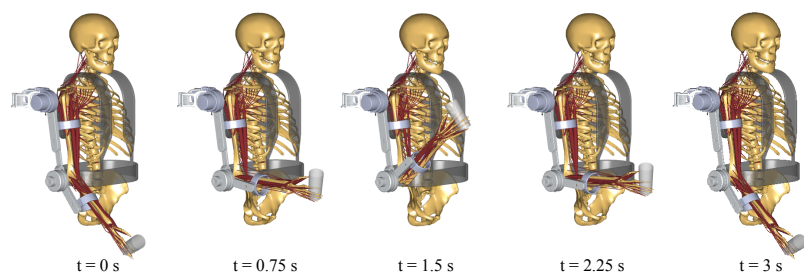

Figure 6: Simulation study of the human-exoskeleton system during the bicep curl

The equivalent human joint torque for each of the degrees of freedom in the arm, are shown in Figs. 7(a)7 (d). As expected, the most loaded joints are shoulder flex/ext (SFE) and elbow flex/ext (EFE), while shoulder abd/add (SAA) and int/ext rotation (SR) are less loaded. Adding the exoskeleton to the simulation leads 
Table 2: Mean muscle activation expressed with equivalent joint torques and maximum muscle activity during biceps curl

\begin{tabular}{clccc}
\hline \hline \multirow{2}{*}{ Motion } & Mean muscle & \multicolumn{3}{c}{ Level of assistance } \\
\cline { 3 - 5 } & activation & w/o Exo & $30 \%$ & $50 \%$ \\
\hline \multirow{2}{*}{ SAA } & Torque [Nm] & 3.44 & 0.65 & -0.53 \\
& Reduction [\%] & - & 81 & 84 \\
\hline \multirow{2}{*}{ SR } & Torque [Nm] & 0.42 & 0.22 & 0.12 \\
& Reduction [\%] & - & 49 & 71 \\
\hline \multirow{2}{*}{ SFE } & Torque [Nm] & 10.62 & 6.36 & 4.41 \\
& Reduction [\%] & - & 40 & 58 \\
\hline \multirow{2}{*}{ EFE } & Torque [Nm] & 8.75 & 4.76 & 3.0 \\
& Reduction [\%] & - & 46 & 66 \\
\hline \multirow{2}{*}{ MMA } & {$[-]$} & 0.38 & 0.26 & 0.25 \\
& Reduction [\%] & - & 30 & 33 \\
\hline \hline
\end{tabular}

to a reduction in all equivalent human joint torques throughout the entire motion. The internal/external rotation of the shoulder experiences a reduction in load, though it is left passive in the exoskeleton. This is caused by the way the human joint torques are calculated, which includes numerous muscle forces surrounding the shoulder glenohumeral joint. Hence, the assistance supplied by the exoskeleton shoulder abb/abd and flex/ext joint indirectly affects the equivalent human joint torque at the shoulder int/ext rotation.

Table 2 lists the mean equivalent human joint torques during the bicep curl. For the low-level assistance, i.e. $30 \%$ assistance, the reduction in muscle activity is in the range of $40-81 \%$, while for the high-level assistance 45-84\%. In both cases, the largest reduction is observed at the shoulder abd/add, where the lowlevel assistance reduced magnitude of the equivalent human joint torque by $81 \%$ and the high-level assistance $(50 \%)$ by $84 \%$.

The maximum muscle activity (MMA) of the muscles in the arm is shown in Fig. 8. The MMA is reduced for both assistance levels, where the largest reduction is seen in the peak value of the MMA. However, the minimum MMA is nearly unaffected. As a result, the mean MMA during the simulation is only slightly reduced from $30 \%$ for the low-level assistance to $33 \%$ for the high-level assistance, respectively.

The energy flow between the human and exoskeleton during the bicep curl is depicted by the muscle work rate of the human arm, as defined in Eq. (3), the power consumption of the active joints in the exoskeleton and the total human energy consumption, as defined in Eq. (4). The human energy consumptions for the three studies are listed in Table 3. The results show that the exoskeleton is able to reduce the energy consumptions with $45 \%$ and $64 \%$ for the low- and

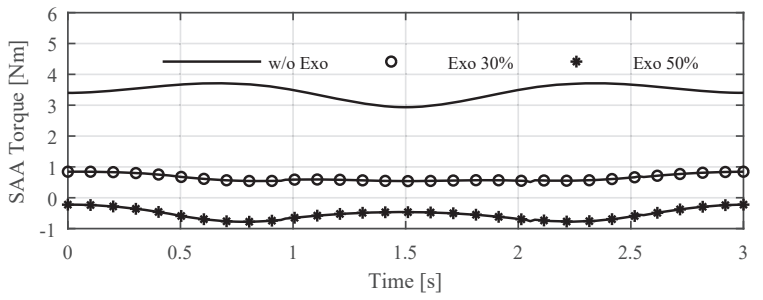

(a)

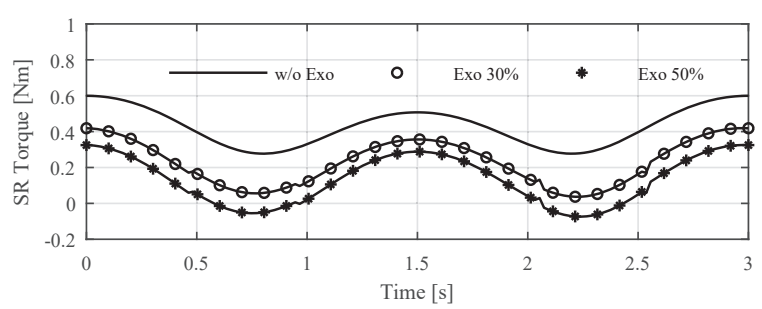

(b)

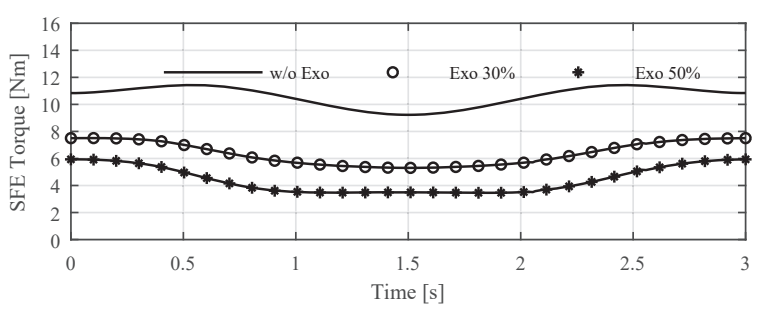

(c)

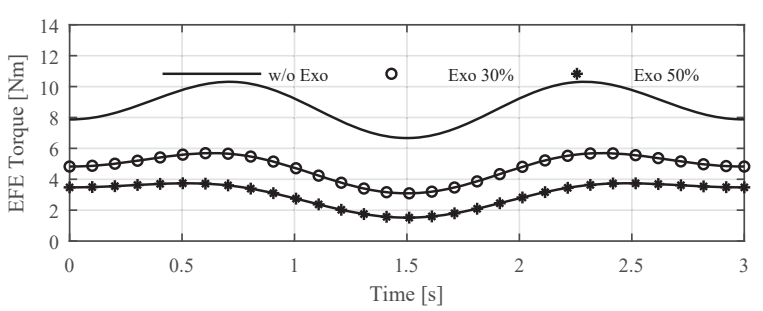

(d)

Figure 7: Equivalent joint torques in the human musculoskeletal system during the bicep curl; (a) shoulder abduction/adduction, (b) shoulder internal/external rotation, (c) shoulder flexion/extension, (d) elbow flexion/extension

high-level assistance, respectively. Hence, the exoskeleton is able to reduce energy consumption satisfactorily according to the control strategy.

In the first half of the simulation, from 0 to $1.5 \mathrm{~s}$, the collective muscles of the upper body carry out concentric work to lift the load, while for the second half of the simulation, from 1.5 to $3 \mathrm{~s}$, the muscles carry out eccentric work to lower the load, see Fig. 9(a). The power consumption of the exoskeleton is mostly related 


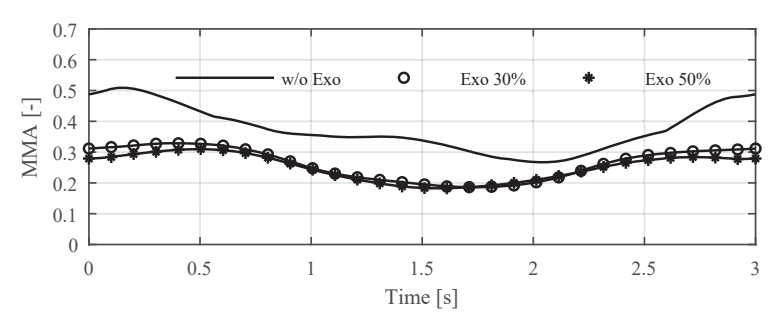

Figure 8: Maximum muscle activity (MMA) in the collective muscles of the upper body during bicep curl

Table 3: Human energy consumption during bicep curl

\begin{tabular}{lccc}
\hline \hline \multirow{2}{*}{ Energy consumption } & \multicolumn{3}{c}{ Level of assistance } \\
\cline { 2 - 4 } & w/o Exo & $30 \%$ & $50 \%$ \\
\hline $\begin{array}{l}\text { Human energy consump- } \\
\text { tion [J] }\end{array}$ & 29.7 & 16.4 & 10.6 \\
$\begin{array}{l}\text { Reduction in energy con- } \\
\text { sumption [\%] }\end{array}$ & - & 45 & 64 \\
\hline \hline
\end{tabular}

to the elbow joint, since both shoulder joints are static, see Figs. 9(b)-9(d). Similarly, as for muscles, the elbow produces positive work in the first half of the simulation and negative work in the second half. Hence, energy is extracted from the human through the exoskeleton electric motors. The design of the electronics of the exoskeleton must be able to handle the excessive energy by either storing it or having it dissipated.

Like the muscle activity, the magnitude of reaction forces in both the shoulder glenohumeral and the elbow joints are reduced when the exoskeleton is assisting the human motion, see Figs. 10(a) and 10(b), with results summarized in Table 4 . The mean reaction force in the shoulder is reduced with $45 \%$ and $64 \%$ for the lowand high-level assistance, respectively, and the mean reaction force in the elbow is reduced with $25 \%$ and $29 \%$, respectively.

Table 4: Magnitude of the mean joint reaction forces during biceps curl

\begin{tabular}{llccc}
\hline \hline \multirow{2}{*}{ Joint } & Mean joint & \multicolumn{3}{c}{ Level of assistance } \\
\cline { 3 - 5 } & reaction force & w/o Exo & $30 \%$ & $50 \%$ \\
\hline \multirow{2}{*}{ Shoulder } & Force [N] & 413.3 & 223.6 & 149.2 \\
& Reduction [\%] & - & 45 & 64 \\
\hline \multirow{2}{*}{ Elbow } & Force [N] & 171.0 & 127.8 & 121.1 \\
& Reduction [\%] & - & 25 & 29 \\
\hline \hline
\end{tabular}

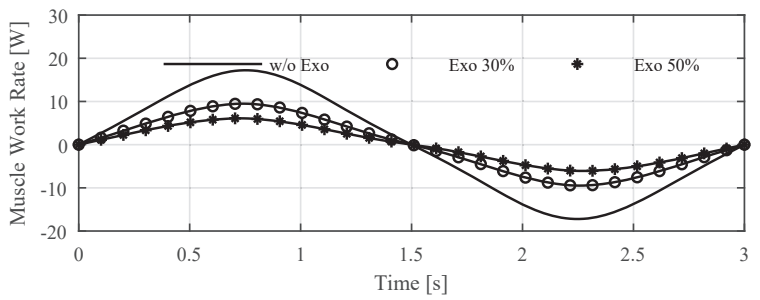

(a)

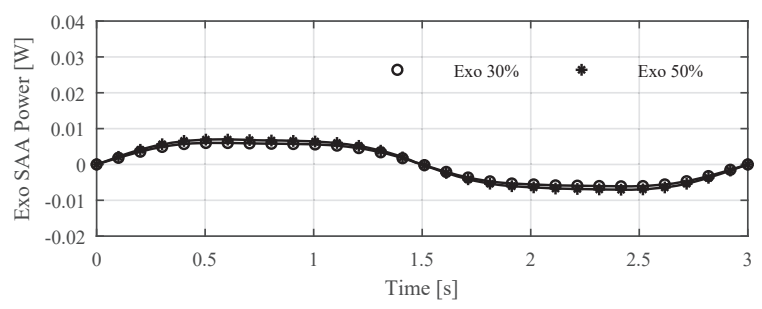

(b)

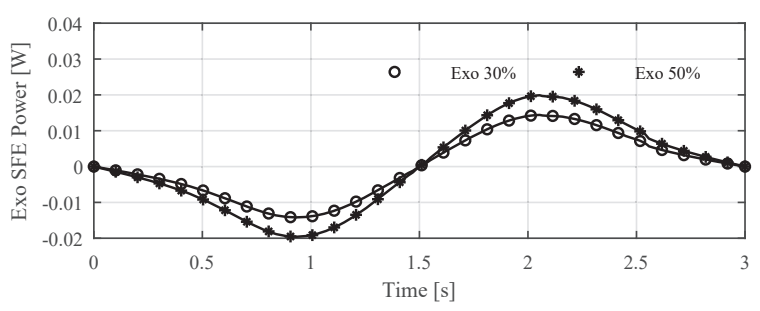

(c)

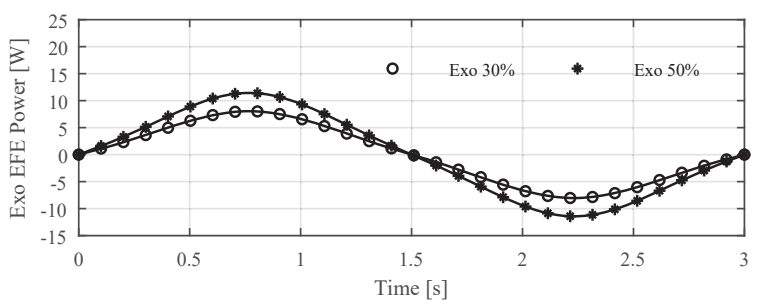

(d)

Figure 9: Energy flow in between the human and exoskeleton during the bicep curl depicted by: (a) the total muscle work rate, (b) the power consumption of the shoulder abb/abd, (c) the power consumption of the shoulder flex/ext, (d) the power consumption of the elbow flex/ext

\subsection{Case 2 - Overhead reach}

In the overhead reach study, a load is lifted from a table to a shelf above shoulder height over a period of 2s. During the lift, the load moved from the outside of the body and across the body, see Fig. 11. This motion requires all degrees of freedom in the shoulder engaged. 


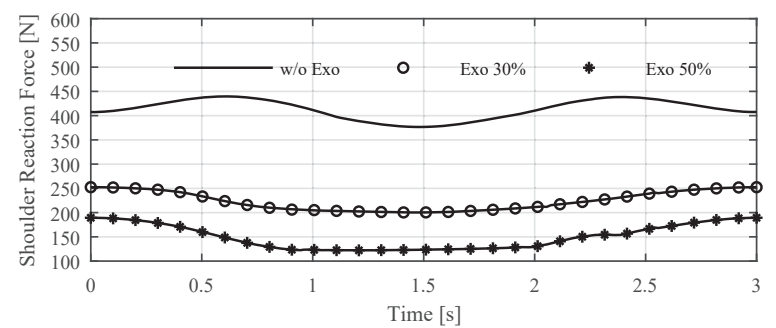

(a)

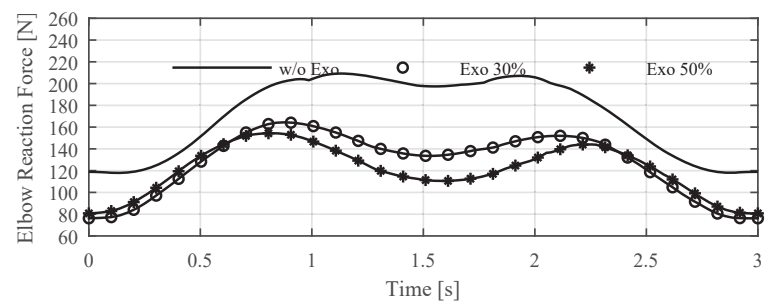

(b)

Figure 10: Magnitude of joint reaction forces in the human musculoskeletal system during the bicep curl; (a) shoulder glenohumeral joint, (b) elbow joint

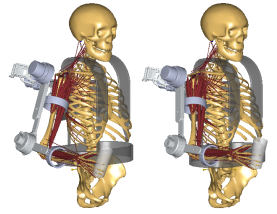

$\mathrm{t}=0 \mathrm{~s}$

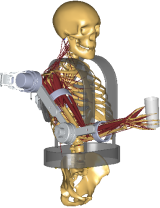

$t=1 s$

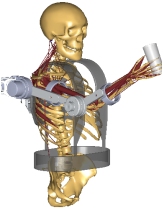

$\mathrm{t}=1.5 \mathrm{~s}$

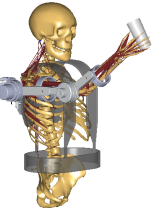

$\mathrm{t}=2 \mathrm{~s}$
Figure 11: Simulation study of the human-exoskeleton system during the overhead reach

The human joint torques are shown in Figs. 12(a)12 (d) and the results are summarized in Table 5 . The most loaded motions are shoulder flex/ext, shoulder abb/abd and elbow flex/ext, while shoulder int/ext rotation is nearly unloaded. Adding the exoskeleton to the simulation leads to a reduction in all assisted human joint torques throughout the entire motion.

The mean muscle activity is reduced with $42 \%$ to $56 \%$ for the low-level assistance case and $61 \%$ to $78 \%$ for the high-level assistance case, respectively. Hence, the mean muscle activity is reduced with slightly more than intended, i.e. $30 \%$ and $50 \%$, respectively. It is also noticed that the mean muscle activity for the shoulder internal/external rotation is increased significantly, with $281 \%$ and $372 \%$, respectively. On the other hand, the torques are still small, which implies that a passive shoulder internal/external rotation is feasible for the

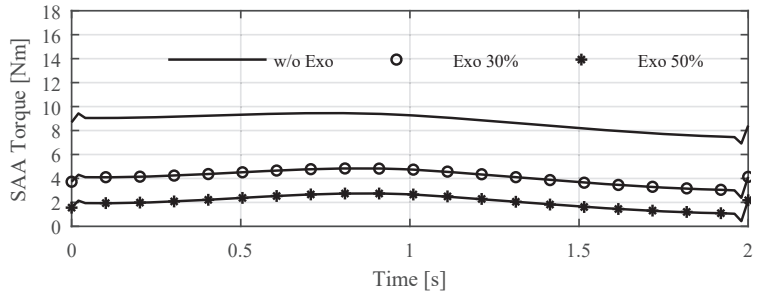

(a)

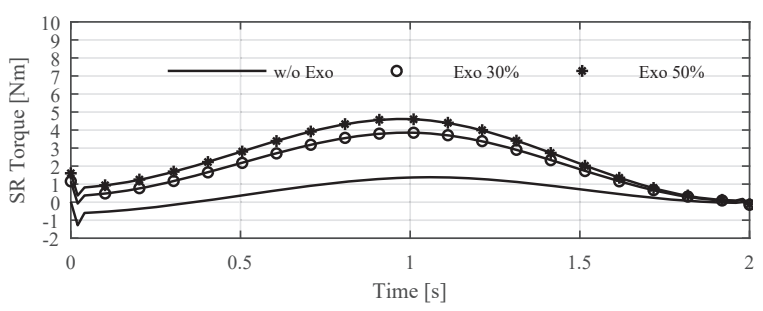

(b)

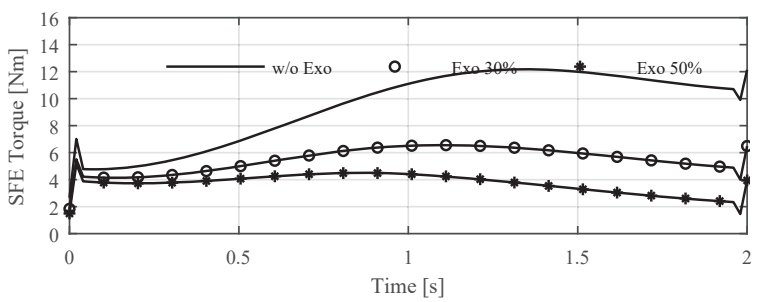

(c)

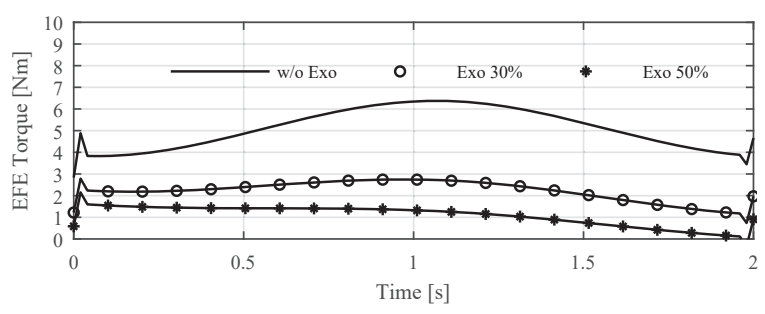

(d)

Figure 12: Equivalent joint torques in the human musculoskeletal system during the overhead reach, (a) shoulder abduction/adduction, (b) shoulder internal/external rotation, (c) shoulder flexion/extension, (d) elbow flexion/extension

assistance of the overhead reach.

The MMA of the muscles in the arm is shown in Fig. 13. The MMA is reduced with the aid of the exoskeleton for both the low-level and high-level assistance. The mean MMA during the simulations, listed in Table 5, is reduced with $45 \%$ in the low-level assistance and $62 \%$ for the high-level assistance.

The human energy consumption for the three sim- 


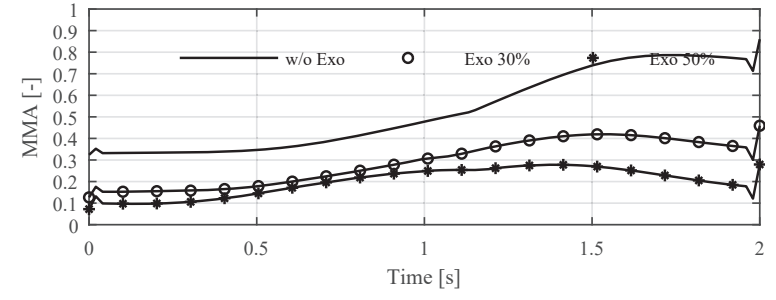

Figure 13: Maximum muscle activity (MMA) in the collective muscles of the upper body during the overhead reach

Table 5: Mean muscle activation expressed with equivalent joint torques and maximum muscle activity during overhead reach

\begin{tabular}{clccc}
\hline \hline \multirow{2}{*}{ Motion } & Mean muscle & \multicolumn{3}{c}{ Level of assistance } \\
\cline { 3 - 5 } & activation & w/o Exo & $30 \%$ & $50 \%$ \\
\hline \multirow{2}{*}{ SAA } & Torque [Nm] & 8.76 & 4.12 & 2.05 \\
& Reduction [\%] & - & 53 & 77 \\
\hline \multirow{2}{*}{ SR } & Torque [Nm] & 0.52 & 1.99 & 2.47 \\
& Reduction [\%] & - & -281 & -372 \\
\hline \multirow{2}{*}{ SFE } & Torque [Nm] & 9.41 & 5.48 & 3.71 \\
& Reduction [\%] & - & 42 & 61 \\
\hline \multirow{2}{*}{ EFE } & Torque [Nm] & 5.09 & 2.21 & 1.08 \\
& Reduction [\%] & - & 56 & 78 \\
\hline \multirow{2}{*}{ MMA } & {$[-]$} & 0.53 & 0.29 & 0.20 \\
& Reduction [\%] & - & 45 & 62 \\
\hline \hline
\end{tabular}

ulations are listed in Table 6. The results show that the exoskeleton is able to reduce the energy consumption with $43 \%$ and $63 \%$ for the low- and high-level assistance, respectively. Hence, the reduction in human energy consumption has a good consistency with the goal of the control strategy.

Throughout the entire overhead reach simulation, the collective muscles of the upper body are carrying out concentric work to lift the load, as shown in Fig. 14(a). Powers associated to motions at each joints are shown in Figs. 14(b)-14(d). It is noted that there exist both positive and negative powers, which imply that energy is both fed and extracted from the exoskeleton to the human. The shoulder and elbow flextion/extension joints both produced positive work to lift the load, while the shoulder abduction/adduction produced negative work to dampen the cross body movement.

The magnitudes of reaction forces in both the shoulder glenohumeral and elbow joint are reduced when the exoskeleton is assisting the human motion, see Figs. 15(a) and 15(b). The magnitude of the mean reaction force in the shoulder is reduced by $36 \%$ and $47 \%$ for the low- and high-level assistance, respectively.

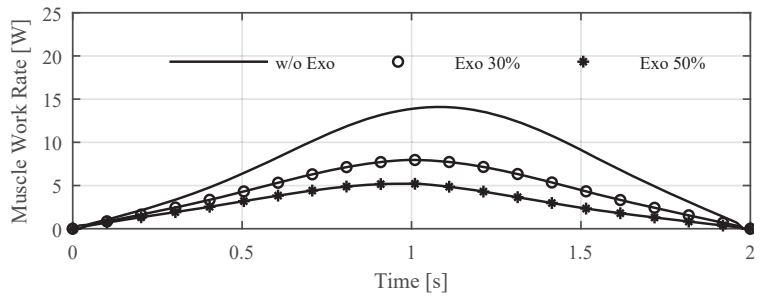

(a)

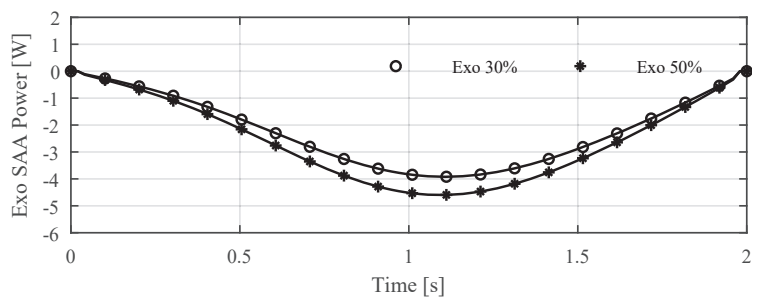

(b)

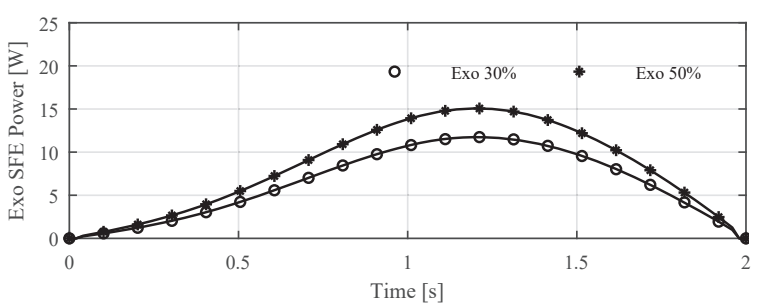

(c)

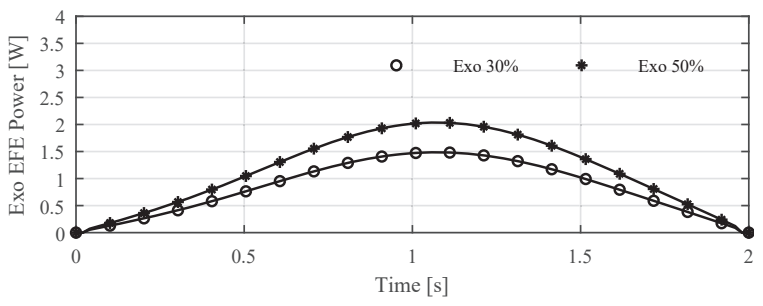

(d)

Figure 14: Energy flow in between the human and exoskeleton during the overhead reach depicted by: (a) the total muscle work rate, (b) the power consumption of the shoulder abb/abd, (c) the power consumption of the shoulder flex/ext, (d) the power consumption of the elbow flex/ext

For the elbow joint, the reduction of the mean reaction force is $53 \%$ and $52 \%$ for the low- and high-level assistance, respectively, as listed in Table 7. 
Table 6: Human energy consumption during overhead reach

\begin{tabular}{lccc}
\hline \hline \multirow{2}{*}{ Energy consumption } & \multicolumn{3}{c}{ Level of assistance } \\
\cline { 2 - 4 } & w/o Exo & $30 \%$ & $50 \%$ \\
\hline $\begin{array}{l}\text { Human energy consump- } \\
\text { tion }[\mathrm{J}]\end{array}$ & 15.3 & 8.7 & 5.6 \\
$\begin{array}{l}\text { Reduction in energy con- } \\
\text { sumption [\%] }\end{array}$ & - & 43 & 63 \\
\hline \hline
\end{tabular}

Table 7: Magnitude of the mean joint reaction forces during overhead reach

\begin{tabular}{llccc}
\hline \hline \multirow{2}{*}{ Joint } & Mean joint & \multicolumn{3}{c}{ Level of assistance } \\
\cline { 3 - 5 } & reaction force & w/o Exo & $30 \%$ & $50 \%$ \\
\hline \multirow{2}{*}{ Shoulder } & Force [N] & 690.3 & 436.0 & 327.1 \\
& Reduction [\%] & - & 36 & 53 \\
\hline \multirow{2}{*}{ Elbow } & Force [N] & 104.2 & 54.9 & 49.8 \\
& Reduction [\%] & - & 47 & 52 \\
\hline \hline
\end{tabular}

\section{Physical construction of UB-AXO}

A prototype of the UB-AXO is built, as shown in Fig. 17 with the design specifications for the upperbody exoskeleton listed in Table 8 . The total weight

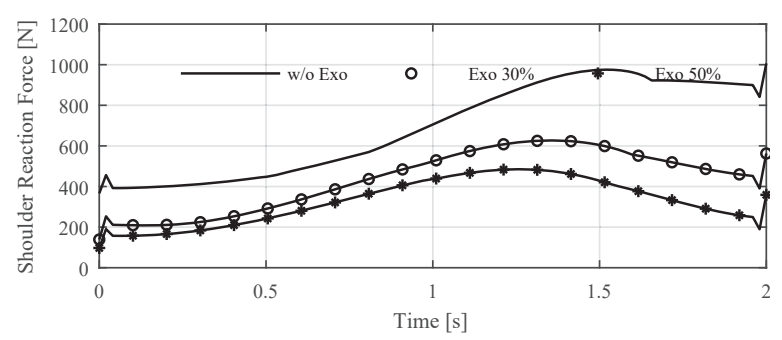

(a)

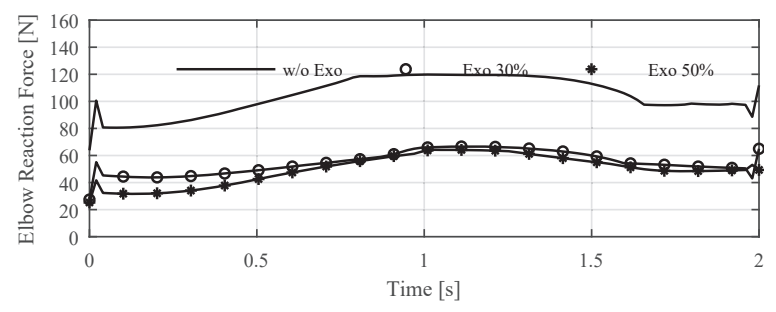

(b)

Figure 15: Magnitude of joint reaction forces in the human musculoskeletal system during the overhead reach, (a) shoulder glenohumeral joint, (b) elbow joint of the UB-AXO is $10 \mathrm{~kg}$ and includes a $3 \mathrm{~kg} \mathrm{Li}$-ion battery, which can power the suit continuously for approximately 4 hours. The prototype will be utilized to test in the physical human-exoskeleton interaction.

Table 8: Actuation of the UB-AXO. Joints 1 and 3 are the active joints in the shoulder mechanism and joint 4 is the elbow joint. Joint 2 is the passive degree of freedom of the DPL

\begin{tabular}{|c|c|c|c|c|}
\hline & Joint 1 & Joint 2 & Joint 3 & Joint 4 \\
\hline $\begin{array}{l}\text { Motor } \\
\text { Type }\end{array}$ & EC-60 & - & EC-60 & EC-45 \\
\hline Gear & LCS- & - & CSD- & CSD- \\
\hline Туре & $17-80$ & & $25-50$ & $25-50$ \\
\hline
\end{tabular}

The upper arm and forearm cuffs are designed based on the simulated contact forces, which must be less than a safe limit. If the contact forces are too high, the use of an exoskeleton may lead to skin irritation and even pressure ulcers Lyder (2003). Excessive skin pressure can compromise both safety and comfort. Regarding safety, the common guideline is to avoid pressures above the ischemic level Lyder (2003); L. Pons (2008), which is the level at which the capillary vessels are no longer able to conduct blood. On the other hand, a comfortable pressure is more complex to define, since it is a highly subjective measure. In Farasyn and Meeusen (2003), a study was conducted on 34 healthy adults to determine the pressure pain threshold (PPT) on selected points on the upper body. The PPT is a measure for the minimum pressure that induces pain or discomfort of human, which can be used to justify whether or not the exoskeleton is comfortable to wear. The PPT of the upper arm is in the range of 83.5 to $96.6 \mathrm{kPa}$ Farasyn and Meeusen (2003). The contact forces in the simulations are linked to contact pressure by estimating the equivalent area that the contact force acts on.

$$
p_{j}^{C}=\frac{f_{j}^{C}}{A_{j}^{C}} \quad A_{j}^{C}=\frac{d \pi w}{12} \quad j=1,2, \ldots, 12
$$

where $p_{j}^{C}$ is the contact pressure of the $j$-the node, $A_{j}^{C}$ is the equivalent area, and $d$ and $w$ are the diameter and width of the cuff, respectively. The diameter of the two cuffs are determined in Section 3.6 as 103 $\mathrm{mm}$ for the upper arm and $76 \mathrm{~mm}$ for forearm. The cuffs are designed to have a width of $150 \mathrm{~mm}$, thus the minimum discomfort pressure force can be determined from Eq. (13) as 337-390 N for the upper arm and 249$288 \mathrm{~N}$ for the forearm. The contact forces in both the biceps curl and overhead reach simulations are shown in Figs. 16(a) to 16(d). The maximum contact forces 
for both upper arm and forearm are below the minimum discomfort pressure forces, which indicates that the exoskeleton is comfortable to wear.

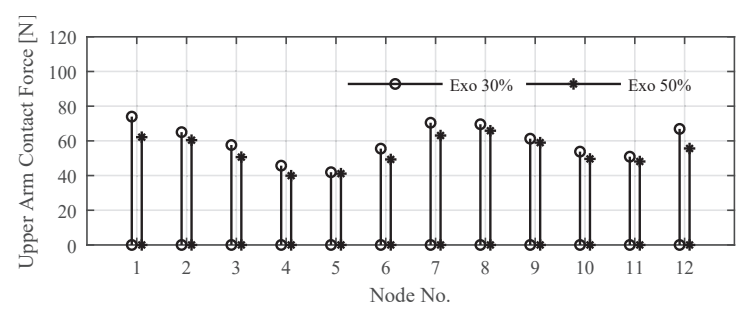

(a)

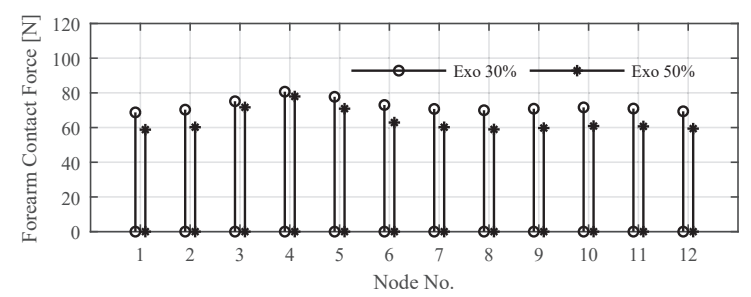

(b)

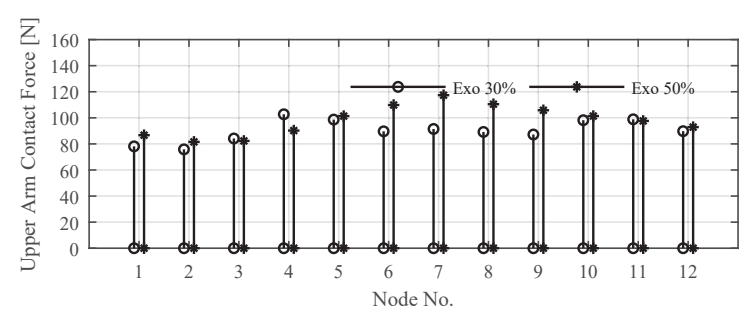

(c)

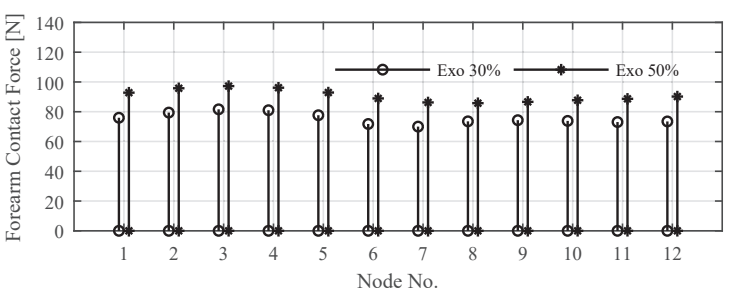

(d)

Figure 16: Maximum normal forces of the contact model nodes in: (a) upper arm cuff in biceps curl study, (b) forearm cuff in biceps curl study, (c) upper arm cuff in overhead study, (d) forearm cuff in overhead study

\section{Discussion and conclusions}

In this paper, a human-exoskeleton model is developed for modeling and analyzing the physical interaction between a human arm and a 4-DoF upper-body ex-

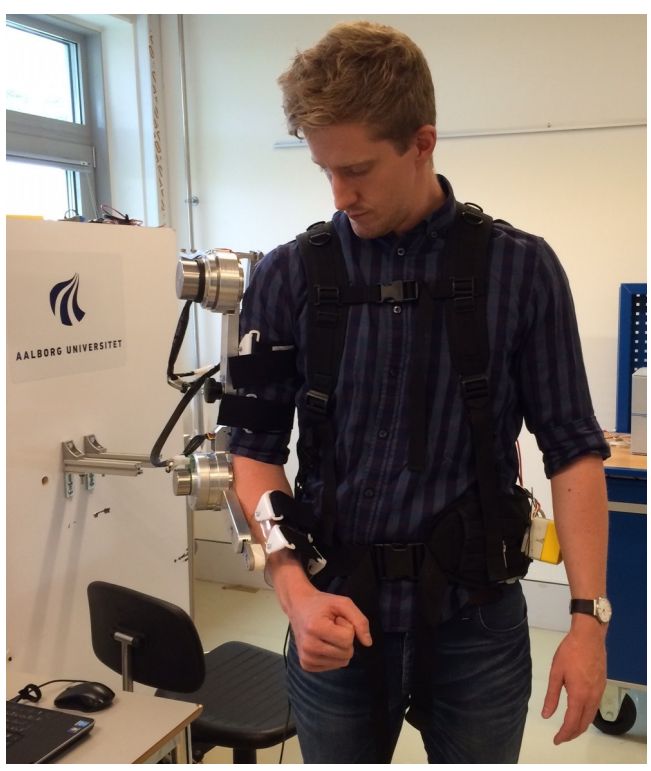

Figure 17: The built prototype of the UB-AXO

oskeleton named UB-AXO. In the model, major factors that affect the physical human-robot interaction are duly considered. They cover the torques and reaction forces at the human joints and the power and energy consumption in the motion assistance. Moreover, the problem of physical contact between the exoskeleton and the human limb is addressed. The model allows us to estimate the dynamic behavior of the humanexoskeleton system and conduct comprehensive simulations to improve our understanding on the pHRI and to finally design an exoskeleton with optimized pHRI.

Two cases were simulated, one for a bicep curl and the other for an overhead reach. The simulation results show that the use of the proposed exoskeleton can significantly reduce both the required maximum muscle activity, targeted human joint torques and muscle energy consumption in the human arm. In our simulated study, the mean maximum muscle activity during the motions was reduced by $30 \%$ to $45 \%$ with the low-level assistance control and $33 \%$ to $62 \%$ for the high-level assistance control. The targeted joint torques, i.e. shoulder abduction/adduction and flexion/extension and elbow flexion/extension, were reduced by $40 \%$ to $81 \%$ with the low-level assistance control and $58 \%$ to $84 \%$ for the high-level assistance control. The largest reductions in joint torques and maximum muscle activity were achieved in static postures, which is in good agreement with the selected control strategy.

The simulation reveals also energy exchange between human and exoskeleton during the physical humanrobot interaction. The energy exchange was studied in terms of instantaneous exoskeleton joint powers and human muscle work rate along with the overall energy 
consumption. In the simulated cases, the human energy consumption was reduced with $45 \%$ and $43 \%$ for the low-level assistance control and $64 \%$ and $63 \%$ for the high-level assistance control in the bicep curl and overhead reach simulation, respectively. The study of energy exchange in pHRI is useful for the development of control strategy and also for the optimum design of the exoskeleton systems.

The simulated cases in this paper include only a bicep curl and overhead reach. With the developed model, more cases of daily activities can be conducted, for example, hand lifting, arm carrying, side reach, etc. On the other hand, experiments of physical humanrobot interaction with the physical exoskeleton system are desirable for comparison with the simulation results. Extra cases of study and experimental investigation are tasks considered in the future.

\section{References}

Agarwal, P., Neptune, R. R., and Deshpande, A. D. A simulation framework for virtual prototyping of robotic exoskeletons. Journal of Biomechanical Engineering, 2016. 138(6):061004. doi:10.1115/1.4033177.

Bai, S. and Rasmussen, J. Modelling of physical human-robot interaction for exoskeleton designs. Proc. of Multibody Dynamics 2011, ECCOMAS Thematic Conference, 2011. (July):1-7.

Bai, S., Virk, G. S., and Sugar, T. G. Wearable exoskeleton systems: Design, control and applications, volume 108. Control, Robotics and Sensors, 2018.

Bhargava, L. J., Pandy, M. G., and Anderson, F. C. A phenomenological model for estimating metabolic energy consumption in muscle contraction. Journal of Biomechanics, 2004. 37(1):81-88. doi:10.1016/S0021-9290(03)00239-2.

Bicchi, A., Peshkin, M. A., and Colgate, J. E. Safety for physical human-robot interaction. Springer Berlin Heidelberg, Berlin, Heidelberg, 2008. doi:10.1007/978-3-540-30301-5_58.

Bock, S. D., Ghillebert, J., Govaerts, R., Elprama, S. A., Marusic, U., Serrien, B., Jacobs, A., Geeroms, J., Meeusen, R., and Pauw, K. D. Passive shoulder exoskeletons: more effective in the lab than in the field? IEEE Transactions on Neural Systems and Rehabilitation Engineering, 2021. 29:173-183. doi:10.1109/TNSRE.2020.3041906.

Christensen, S. and Bai, S. Kinematic analysis and design of a novel shoulder exoskeleton using a double parallelogram linkage. Journal of Mechanisms and Robotics, 2018. 10(4). doi:10.1115/1.4040132.

Davis, K. G., Reid, C. R., Rempel, D. D., and Treaster, D. Introduction to the human factors special issue on user-centered design for exoskeleton. Human Factors, 2020. 62(3):333-336. doi:10.1177/0018720820914312.

De Santis, A., Siciliano, B., De Luca, A., and Bicchi, A. An atlas of physical humanrobot interaction. Mechanism and Machine Theory, 2008. 43(3):253-270. doi:10.1016/j.mechmachtheory.2007.03.003.

Farasyn, A. and Meeusen, R. Pressure pain thresholds in healthy subjects: influence of physical activity, history of lower back pain factors and the use of endermology as a placebo-like treatment. Journal of Bodywork and Movement Therapies, 2003. 7(1):5361. doi:10.1016/S1360-8592(02)00050-5.

Gopura, R., Bandara, D., Kiguchi, K., and Mann, G. Developments in hardware systems of active upper-limb exoskeleton robots: A review. Robotics and Autonomous Systems, 2016. 75:203220. doi:10.1016/j.robot.2015.10.001.

Gull, M. A., Bai, S., and Bak, T. A review on design of upper limb exoskeletons. Robotics, 2020. 9(1). doi:10.3390/robotics9010016.

Heinzmann, J. and Zelinsky, A. Quantitative safety guarantees for physical human-robot interaction. The International Journal of Robotics Research, $2003 . \quad 22(7-8): 479-504$. doi:10.1177/02783649030227004.

L. Pons, J. Wearable robots: biomechatronic exoskeletons. Wiley, 2008.

Lyder, C. H. Pressure ulcer prevention and management. JAMA, 2003. 289(2):223-226. doi:10.1001/jama.289.2.223.

Narayanan, M., Kannan, S., Mendel, F., and Krovi, V. Case studies of musculoskeletal-simulationbased rehabilitation program evaluation. IEEE Transtions on Robotics, 2009. 25(3):634-638. doi:10.1109/TRO.2009.2019780.

Nf, M. B., Junius, K., Rossini, M., RodriguezGuerrero, C., Vanderborght, B., and Lefeber, D. Misalignment compensation for full humanexoskeleton kinematic compatibility: State of the art and evaluation. Applied Mechanics Reviews, 2019. 70(5). doi:10.1115/1.4042523. 
Pacifico, I., Scano, A., Guanziroli, E., Moise, M., Morelli, L., Chiavenna, A., Romo, D., Spada, S., Colombina, G., Molteni, F., Giovacchini, F., Vitiello, N., and Crea, S. An experimental evaluation of the proto-MATE: A novel ergonomic upper-limb exoskeleton to reduce workers' physical strain. IEEE Robotics Automation Magazine, 2020. 27(1):54-65. doi:10.1109/MRA.2019.2954105.

Peebles, L. and Norris, B. Adultdata: The handbook of adult anthropometric and strength measurements: Data for design safety. Government consumer safety research. Department of Trade and Industry, 1999. doi:10.1177/106480469900700310.

Rasmussen, J., Damsgaard, M., and Voigt, M. Muscle recruitment by the $\min / \max$ criterion a comparative numerical study. Journal of Biomechanics, 2001. 34(3):409-415. doi:10.1016/S0021-9290(00)00191-3.

Rowe, M. F. Safety measures for conducting exercise oxygen consumption, VO2, tests in developing countries. Tropical Doctor, 2020. 50(3):280-281. doi:10.1177/0049475520918033.

Schiele, A. An explicit model to predict and interpret constraint force creation in pHRI with exoskeletons. In 2008 IEEE International Conference on Robotics and Automation. pages 1324-1330, 2008. doi:10.1109/ROBOT.2008.4543387.

Schiele, A. and van der Helm, F. C. T. Kinematic design to improve ergonomics in human machine interaction. IEEE Transactions on Neural Systems and Rehabilitation Engineering, 2006. 14(4):456469. doi:10.1109/TNSRE.2006.881565.

Skals, S., Jung, M. K., Damsgaard, M., and Andersen, M. S. Prediction of ground reaction forces and moments during sports-related movements. Multibody System Dynamics, 2016. 39(3):175-195. doi:10.1007/s11044-016-9537-4.

Sylla, N., Bonnet, V., Colledani, F., and Fraisse, P. Ergonomic contribution of ABLE exoskeleton in automotive industry. International Journal of Industrial Ergonomics, 2014. 44(4):475-481. doi:10.1016/j.ergon.2014.03.008.

Winter, D. A. Biomechanics and Motor Control of Human Movement. JOHN WILEY \& SONS, INC., Hoboken, New Jersey, 2008. doi:10.1002/9780470549148.

Zhou, L., Bai, S., Andersen, M. S., and Rasmussen, J. Modeling and design of a spring-loaded, cabledriven, wearable exoskeleton for the upper extrem- ity. Modeling, Identification and Control, 2015. 36(3):167-177. doi:10.4173/mic.2015.3.4.

Zhou, L., Li, Y., and Bai, S. A human-centered design optimization approach for robotic exoskeletons through biomechanical simulation. Robotics and Autonomous Systems, 2017. 91:337-347. doi:10.1016/j.robot.2016.12.012.

\section{Appendix A}

Entries of the Jacobian matrix of Eq. (5):

$$
\begin{aligned}
J_{11}= & -L_{f} s \theta_{4}\left(c \theta_{1} c \theta_{3}-c \theta_{2} s \theta_{1} s \theta_{3}\right)-L_{u} c \theta_{1} s \theta_{3} \\
& -L_{f} c \theta_{4}\left(c \theta_{1} s \theta_{3}+c \theta_{2} c \theta_{3} s \theta_{1}\right)-L_{u} c \theta_{2} c \theta_{3} s \theta_{1} \\
J_{12}= & -c \theta_{1} s \theta_{2}\left(L_{f} c\left(\theta_{3}+\theta_{4}\right)+L_{u} c \theta_{3}\right) \\
J_{13}= & L_{f} s \theta_{1} s \theta_{3} s \theta_{4}-L_{f} c \theta_{3} c \theta_{4} s \theta_{1}-L_{u} c \theta_{1} c \theta_{2} s \theta_{3} \\
& -L_{u} c \theta_{3} s \theta_{1}-L_{f} c \theta_{1} c \theta_{2} c \theta_{3} s \theta_{4}-L_{f} c \theta_{1} c \theta_{2} c \theta_{4} s \theta_{3} \\
J_{14}= & L_{f} s \theta_{1} s \theta_{3} s \theta_{4}-L_{f} c \theta_{3} c \theta_{4} s \theta_{1}-L_{f} c \theta_{1} c \theta_{2} c \theta_{3} s \theta_{4} \\
& -L_{f} c \theta_{1} c \theta_{2} c \theta_{4} s \theta_{3} \\
J_{21}= & L_{u} c \theta_{1} c \theta_{2} c \theta_{3}-L_{f} c \theta_{4}\left(s \theta_{1} s \theta_{3}-c \theta_{1} c \theta_{2} c \theta_{3}\right) \\
& -L_{u} s \theta_{1} s \theta_{3}-L_{f} s \theta_{4}\left(c \theta_{3} s \theta_{1}+c \theta_{1} c \theta_{2} s \theta_{3}\right) \\
J_{22}= & -s \theta_{1} s \theta_{2}\left(L_{f} c\left(\theta_{3}+\theta_{4}\right)+L_{u} c \theta_{3}\right) \\
J_{23}= & L_{u} c \theta_{1} c \theta_{3}+L_{f} c \theta_{1} c \theta_{3} c \theta_{4}-L_{f} c \theta_{1} s \theta_{3} s \theta_{4} \\
& -L_{u} c \theta_{2} s \theta_{1} s \theta_{3}-L_{f} c \theta_{2} c \theta_{3} s \theta_{1} s \theta_{4} \\
& -L_{f} c \theta_{2} c \theta_{4} s \theta_{1} s \theta_{3} \\
J_{24}= & L_{f} c \theta_{1} c \theta_{3} c \theta_{4}-L_{f} c \theta_{1} s \theta_{3} s \theta_{4}-L_{f} c \theta_{2} c \theta_{3} s \theta_{1} s \theta_{4} \\
& -L_{f} c \theta_{2} c \theta_{4} s \theta_{1} s \theta_{3} \\
J_{31}= & 0 \\
J_{32}= & -c \theta_{2}\left(L_{f} c\left(\theta_{3}+\theta_{4}\right)+L_{u} c \theta_{3}\right) \\
J_{33}= & s \theta_{2}\left(L_{f} s\left(\theta_{3}+\theta_{4}\right)+L_{u} s \theta_{3}\right) \\
J_{34}= & L_{f} s\left(\theta_{3}+\theta_{4}\right) s \theta_{2} \\
J_{41}= & 0 \\
J_{42}= & -s \theta_{1} \\
J_{43}= & c \theta_{1} s \theta_{2} \\
J_{64}= & c \theta_{1} s \theta_{2} \\
J_{51}= & 0 \\
J_{52}= & c \theta_{1} \\
J_{53}= & s \theta_{1} s \theta_{2} \\
J_{54}= & s \theta_{1} s \theta_{2} \\
J_{61}= & 0 \\
J_{64} & \\
& \\
J_{63} & \\
&
\end{aligned}
$$

